\title{
BARRANCOS Y RAMBLAS: SU INCORPORACIÓN AL ENTRAMADO URBANO EN EL SURESTE PENINSULAR*
}

\author{
Margarita Box Amorós \\ Alfredo Morales Gil
}

\begin{abstract}
RESUMEN
El crecimiento urbano de los núcleos de población instalados sobre glacis y conos de deyección, en el sureste peninsular, se realizó, en múltiples ocasiones, incorporando a la trama urbana cauces de barrancos y ramblas que únicamente se muestran funcionales con ocasión de aguaceros de cierta intensidad. Frente a la adecuación, en épocas pasadas, del nuevo entramado urbano a estas arterias, reflejo del absoluto respeto y clara conciencia de su naturaleza, se asiste hoy, a una total inadaptación de la ciudad a las condiciones de escorrentía, con los graves problemas que ello genera cuando se producen chubascos copiosos que hacen recobrar a estos viales su funcionamiento real.
\end{abstract}

\section{SUMMARY}

The urbano growth of settlements located on debris and deyecta, in the Peninsular Southeast, was often carried out, using, as part of the urban structure, ravines and gullies which became operative only in case of highly intensive rains. In clear contrast to the past adaptation of the new urban structure to the conditions imposed by the water beds, due to the respect and clear awareness of their very nature, we are confronted, nowadays with the total inadaptation of the towns to said conditions. This entails a series of serious problems which appear whenever these water beds are recovering their function because of heave rainfalls.

Buena parte de los núcleos de población del sureste peninsular se situaron, en origen, sobre glacis y conos de deyección, superficies que efectúan la transición entre la montaña propiamente dicha y la llanura inundable, en una posición destacada del relieve, buscando un emplazamiento que, además de defensivo, fuera, a la vez, capaz de salvaguardar a la

* Este artículo procede del Proyecto de Investigación sobre «Insuficiencia de recursos hídricos y riesgo de avenidas en las regiones de Valencia y Murcia», patrocinado por la Comisión Interministerial de Ciencia y Tecnología. 
población de la arroyada e inundación de las zonas bajas. No obstante, sobre las mismas discurren ramblas y barrancos que avenan las superficies montañosas y que, por las propias características topográficas, edáficas y climáticas de la zona, forman redes de drenaje muy ramificadas. La aleatoriedad de la circulación hídrica de estos aparatos fluviales ha motivado que, en múltiples ocasiones, aquéllos que discurrían próximos a núcleos de población, hayan sido incorporados a la trama urbana, con el crecimiento de los mismos, y sus lechos transformados en simples viales.

En un primer momento, la proximidad de uno o más barrancos al núcleo original de la población representaba un elemento importante desde el punto de vista defensivo, al actuar muchas veces como foso natural o de la muralla; así, la ciudad de Almería se amplió en el s. XI con un arrabal a oriente y otro a occidente del núcleo antiguo y sus límites extremos fueron sendos barrancos aprovechados como fosos naturales. Eliminada esa necesidad e iniciado el crecimiento demográfico, que demanda suelo para el asentamiento de la población, barrancos y ramblas han sido, paulatinamente integrados al entramado urbano, una circunstancia que ha dejado su impronta en la morfología de los distintos núcleos y en diferentes detalles constructivos de las viviendas situadas en sus márgenes.

Esa incorporación paulatina de estas redes de avenamiento se hizo siempre respetando los lechos originales, e incluso el nuevo callejero creado recibe, en muchos casos, denominaciones alusivas a la naturaleza física de los mismos. En efecto, en las provincias de Alicante, Murcia y Almería, se han contabilizado más de 500 vías urbanas que aluden, de forma expresiva, a su condición de antiguas arterias fluviales, o lugar de paso de algún tipo de infraestructura relacionada con el regadío eventual de turbias (Vid. Apéndice).

Abundante resulta, en este sentido, el término «barranco» que, en ocasiones, da de manera individualizada nombre a una calle, mientras en otras va acompañado de una segunda denominación, por ejemplo «Calle Barranco del Caño» en Tabernas (Almería), «Calle Barranco de Amorós», en Crevillente (Alicante) o «Calle Barranco de Las Cruces» en Puerto Lumbreras (Murcia). Se utilizan, asimismo, derivaciones del vocablo «barranco», tales como «barranquete» — «Calle Barranquete» en La Alberca (Murcia), o «Calle Barranquete» en Illar (Almería)—, o su variante catalana «barranquet» (diminutivo de «barranc» — «Calle Barranquet» en Villajoyosa, Orcheta, Muro de Alcoy, Bañeres, entre otras, todas ellas en tierras alicantinas-; el término «barranquillo» se ha registrado únicamente en poblaciones de Almería y Murcia (Velefique, Lucen y San José, en la primera provincia indicada, y en Torre Pacheco, en la segunda); igualmente se ha anotado el término «barrancal» o «barrancar», que da nombre a una calle en la población de Mula (Murcia) y que indica, según el diccionario de la Real Academia de la Lengua Española, aquel «sitio donde hay muchos barrancos».

Mayoría constituye el término «rambla» que, al igual que en el caso del vocablo «barranco», aparece como denominación única o conjuntamente con un topónimo que contribuye a personalizar la arteria; la relación de calles, en ambos casos es extensa y se reparte por la geografía de las tres provincias analizadas. También se utilizan derivaciones del término como «rambleta» (en Cox, La Cañada y Elche, en la provincia de Alicante; y en Almagros y Jimenado en la de Murcia); diminutivo con terminación propia del habla murciana es el de «ramblica» que da nombre a dos calles en las localidades de Calasparra y Totana (Murcia); exclusivo también de esta provincia es el vocablo «ramblizo» que hace referencia al «sitio por donde corren las aguas de los turbiones y avenidas», y que es utilizado en Yecla y El Bojar-Beniaján; en Almería y Murcia se utiliza, igualmente, el diminutivo «ramblilla», con ejemplos en Seron, Las Norias y Bayarcal (Almería) y en 
Cartagena y Alhama de Murcia (Murcia), si bien, en algún caso, a dicho término acompaña una denominación más específica como «Ramblilla de Híjar» en Laujar de Andarax (Almería), «Ramblilla de Tejeras» y «Ramblilla de San Lázaro» en Lorca (Murcia). En dos localidades, ambas en la provincia de Murcia, Alhama de Murcia y Totana, se ha registrado el topónimo «ramblar» que, según el diccionario de la Real Academia de la Lengua Española, se refiere a «aquel lugar en el que confluyen varias ramblas».

Alusivo también al tipo de cauce es el término «torrentera» que, en Calasparra (Murcia) da nombre a una calle; dicho vocablo es sinónimo de cauce de un torrente o «corriente impetuosa de aguas que sobreviene en tiempos de muchas lluvias».

El término «boquera» o «boquerón», se utiliza también como denominación de algunos viales, caso, por ejemplo de la calle de la Boquera del Calvario en Crevillente; se trata de un término que alude a un elemento del regadío, es decir, un canal por el que se desvían, hasta los campos de cultivo, aguas de ramblas y barrancos, con ocasión de precipitaciones copiosas e intensas. El crecimiento urbano en algunos núcleos supuso la ocupación de 
GráfICo 2. Plano de Alicante, copia del dibujado por Manuel Miralles en 1794.

1. Barranco de Canicia; 2. Barranco de San Blas. Ambos cursos utilizados, en origen como fosos de las murallas, fueron después incorporados a la trama urbana y sus cauces naturales difuminados por edificaciones y viales.

parte de los alrededores cultivados, pero se mantuvo el trazado y funcionalidad de las boqueras, de las que seguían beneficiándose predios inferiores, creando, por tanto, una servidumbre de paso que fue respetada.

La adaptación de esas arterias fluviales a la nueva trama urbana tiene características diferentes dependiendo del mayor o menor encajamiento del barranco; en aquéllos de pronunciada incisión, se respeta el trazado original del curso, que condiciona así el desarrollo urbano de sus márgenes; por el contrario, barrancos y ramblas escasamente encajados, son, en ocasiones, manipulados ante el crecimiento urbano, de forma que sus lechos son desviados hacia la parte externa de los núcleos; no obstante, esa modificación del trazado original encuentra límites y no resulta extraño que la red urbana de traza poligonal implantada, en algunas poblaciones, desde el s. XVIII se vea, en cierta manera, alterada por arterias diagonales que no son sino la adaptación a la nueva morfología urbana de barrancos y ramblas, mientras que el derrame de los mismos en los ensanches del s. XIX y principios del s. XX, queda prácticamente difuminado, claro indicio de la progresiva pérdida de conciencia por parte del hombre de la auténtica naturaleza de estas «calles». Sirva como ejemplo el gráfico 1 perteneciente a la localidad de Jumilla y en el que las rambletas que descienden desde el Cerro del Castillo en dirección sur, fueron desviadas 
Fото 1. "Calle Barranco» (Gérgal-Almería), en la que buena parte de las edificaciones recayentes a la misma son fachadas traseras.

hacia el E. por las planificaciones del s. XVIII, desapareciendo de la morfología urbana en los ensanches del s. XIX.

En algunos casos, estas vías tan peculiares han desempeñado, incluso hasta mediados del s. XX, el papel de colectores de aguas residuales, es decir, albañales, pues, como afirma Quirós «...en muchas ciudades la carencia de aguas corrientes unida a la precariedad de las lluvias y a su irregularidad estacional, reducía la capacidad de arrastre de los albañales, en los que fácilmente se estancaban los detritus hasta que un aguacero les daba salida...» ${ }^{1}$. Es por ello que, ante la ausencia de alcantarillado, las viviendas instaladas en sus márgenes aportaran, mediante atarjeas, las aguas residuales a estas arterias que disponían de la pendiente suficiente para su evacuación. A tenor de ello, resulta frecuente que sea la parte posterior de la vivienda la que de vista a la calle-rambla, alejando así, al máximo posible, los focos de infección y los riesgos de inundación (Fot. 1).

Otra particularidad que diferencia estas «calles» y que guarda estrecha relación con su funcionamiento natural es la forma de la calzada; así, frente a la disposición convexa que presentan habitualmente los viarios urbanos, éstas adquieren forma cóncava al objeto de concentrar la arroyada en la parte central y evitar, en lo posible, la erosión de las márgenes (Fot. 2). Esta característica se patentiza de forma más contundente cuando se procede al empedrado, adoquinamiento o asfaltado de las mismas; e incluso, en ocasiones, la parte central de la calzada se reviste de un material distinto al del resto y de mayor consistencia (Fot. 3).

Cuando es la fachada principal de las viviendas la que recae sobre la rambla, los portales de acceso suelen estar elevados entre 0,50 y $1 \mathrm{~m}$ sobre la rasante de la misma 
Fото 2. «Calle Rambleta del Convento» (Jumilla-Murcia). El perfil transversal de la calzada muestra una disposición cóncava al objeto de concentrar la arroyada en su parte central y evitar la erosión de las márgenes.

(Fot. 4), e incluso en aquellos lugares donde era posible una crecida por encima de estos umbrales, dichos portales estaban provistos en sus extremos de sólidas piedras de sillería con ranuras labradas en las que se introducía un madero o tablacho, cuya sujeción quedaba reforzada en los extremos con yeso; se conseguía así impedir la entrada de las aguas circulantes en el interior de las viviendas (Fot. 5).

Las soluciones adoptadas en los diferentes núcleos que acogen en su seno arterias fluviales de funcionamiento intermitente y espasmódico, para reducir, en la medida de lo posible, los negativos efectos de la circulación de las aguas, son variadas; algunos ejemplos pueden ilustrar estas adaptaciones. En la llamada «Calle Barranco del Caño» en Tabernas (Almería), el problema se ha resuelto respetando la topografía original, de forma que dicha calle discurre a menor cota que las circundantes, instaladas sobre las primitivas márgenes de dicho barranco, al tiempo que para evitar la desconexión entre una y otra parte del cauce cuando se producen precipitaciones abundantes y la calle recobra su funcionamiento original, se ha instalado una pasarela que permite salvar el obstáculo en situaciones de esta naturaleza (Fot. 6). Adecuaciones menos espectaculares, pero no por ello sin interés, las encontramos en otras arterias, como en la «Calle La Rambla» en Garrucha (Almería), donde el primitivo cauce, transformado en viario urbano y atravesado por calles perpendiculares, cuenta, en su tramo bajo, con una acera badenada que permite el paso del agua y evita la inundación en ese sector del núcleo (Fot. 7). 
Fото 3. «Calle Rambla del Pozo» (Carboneras-Almería). La disposición cóncava de la calzada se refuerza con un canalillo central de fábrica más sólida.

Fото 4. "Calle Barranco» (Gérgal-Almería). Portal de vivienda sobreelevado respecto a la rasante de la calzada y escaleras de acceso al mismo. 
Fото 5. «Calle Rambleta del Pósito» (Jumilla-Murcia). Portalón con piedras de sillería que presentan ranuras inclinadas para la introducción de un tablacho que evite la penetración de las aguas de lluvia.

En alguna ocasión, el lecho natural ha sido respetado en su integración en el casco urbano, y las únicas modificaciones se refieren al encauzamiento mediante un canal y ajardinamiento de sus márgenes, como es el caso de la llamada «Calle del Badén de Rico Lucas» en Pinoso (Alicante) (Fot. 8). Mencionar también el llamado «Parque de Ruiz Hidalgo» (Murcia) que, hasta su desaparición en 1955-56, experimentaba la inundación del Segura, si bien éste era un hecho completamente asumido por los habitantes de la ciudad.

Cursos autóctonos, de funcionalidad intermitente, han sido, pues, artificializados y sus márgenes ocupadas por el desarrollo urbano de muchos núcleos del sureste peninsular. Ahora bien, la consideración de estos elementos del medio natural como recursos o como amenazas para la organización del territorio depende, las más de las veces, del desarrollo del sistema socioeconómico y del nivel técnico de los diferentes grupos humanos; no 
Fото 6. «Calle Barranco del Caño» (Tabernas-Almería). Esta vía urbana discurre a menor cota que las adyacentes, conservando la topografía original del cauce del barranco. Al fondo se observa una pasarela que cruza el cauce y une las calles inmediatas y a mayor altura.

obstante, las consecuencias de esa interferencia dependen, en gran medida, del nivel que alcancen las modificaciones, siendo negativas cuando se sobrepasan los límites de tolerancia del medio natural.

Precisamente en esta última línea se enmarcan las transformaciones que, desde los años cincuenta del s. XX, se han producido en muchas poblaciones del sureste español. La ordenación de barrancos y ramblas que atraviesan núcleos urbanos no suele ser objeto de determinaciones en los documentos del planeamiento, sobre todo, cuando se trata de arterias que no afectan sólo al perímetro urbano, y sobre las que deben primar ordenaciones de tipo supramunicipal. En efecto, la planificación urbanística ha ignorado, en unos casos, el trazado de barrancos y ramblas, al considerarlos como algo habitual en la percepción de la ciudad, máxime cuando su funcionalidad posee carácter intermitente. Los ejemplos a enumerar serían abundantes, pero sirva como muestra la actual Rambla de Méndez Núñez, en la ciudad de Alicante, que constituye el tramo final del llamado Barranco de Canicia, cuyo trazado original prácticamente ha desaparecido de la morfología urbana, pero que recobra totalmente su funcionalidad cuando se producen chubascos copiosos e intensos, provocando, así, la inundación de la parte baja de la ciudad, al estar su desembocadura obstruida por el Paseo de la Explanada, dispuesto en paralelo a la línea de costa; en otros casos, se ha pretendido su canalización, en no pocas ocasiones, subterránea, que resulta casi siempre insuficiente, cuando se producen precipitaciones torrenciales, para el avenamiento de considerables volúmenes, que, además, se han incrementado por la impermeabilización que supone el asfaltado de las calles. Es el caso, entre otros, de la localidad de 
Fото 7. «Calle La Rambla» (Garrucha-Almería). Una solución sencilla que muestra la adaptación del viario urbano a las condiciones naturales es el badenado de esta acera que permite la evacuación de la arroyada de la rambla.

Pedreguer, donde el crecimiento urbano se produjo haciendo tabla rasa del Barranc dels Poets que lo atravesaba de N. a S. y cuyo cauce aparece hoy encajonado y zigzageante, con repetidos cruces de calles y de tuberías que reducen la rapidez de descarga del caudal acumulado.

En definitiva, el desarrollo económico y urbanístico de muchas localidades del sureste peninsular se ha realizado ignorando las características del medio físico y ello ha traído como consecuencia la creación de puntos conflictivos cuya solución se aborda, las más de las veces, después de producidas auténticas situaciones catastróficas. 
Fото 8. "Calle Badén de Rico Lucas» (Pinoso-Alicante), donde el barranco ha sido respetado en su trazado y canalizado con muretes en sus márgenes.

\section{Bibliografía}

CAPEL MOLINA, J. J.: Riesgos naturales en la provincia de Almería, Inst. de Estudios Almerienses de la Diputación de Almería, 1991.

GÓMEZ CRUZ, M.: Morfología urbana de Almería en el siglo XVIII, Edit, Centro de Estudios Municipales y de Cooperación Interprovincial, Granada, 1990.

LARA VALLE, J. J.: Desarrollo y crisis urbana en Almería (1900-1980), Edit. Cajal, Almería, 1989. MORALES GIL, A.: El Altiplano de Jumilla-Yecla, Univ. de Murcia, Murcia, 1972.

QUIROS LINARES, F. «El abasto de aguas y la limpieza pública en las ciudades españolas a mediados del s. XIX», en Los paisajes del agua, Edit. Univ. de Alicante y Univ. de Valencia, 1989.

- Las ciudades españolas en el s. XIX, Edit. Ambito, Valladolid, 1991. 


\begin{tabular}{|c|c|c|}
\hline Calle & Población & Provincia \\
\hline AGUADA & AGUA AMARGA & ALMERÍA \\
\hline AGÜERA & ALGAR & MURCIA \\
\hline ARROYO & JAVALÍ NUEVO & MURCIA \\
\hline ARROYO & LA ÑORA & MURCIA \\
\hline ARROYOS, LOS & ALBUJÓN & MURCIA \\
\hline BARRANCAL & MULA & MURCIA \\
\hline BARRANCAR & MULA & MURCIA \\
\hline BARRANCO & ESPINARDO & MURCIA \\
\hline BARRANCO & HURCHILLO & ALICANTE \\
\hline BARRANCO & PUERTO LUMBRERAS & MURCIA \\
\hline BARRANCO & FORTUNA & MURCIA \\
\hline BARRANCO & STA. MARÍA DEL ÁGUILA & ALMERÍA \\
\hline BARRANCO & MACAEL & ALMERÍA \\
\hline BARRANCO & FÉLIX & ALMERÍA \\
\hline BARRANCO & TOTANA & MURCIA \\
\hline BARRANCO & GORGAL & ALMERÍA \\
\hline BARRANCO & ATOCHARES & ALMERÍA \\
\hline BARRANCO & LORQUÍ & MURCIA \\
\hline BARRANCO & CARTAGENA & MURCIA \\
\hline BARRANCO & INSTINCIÓN & ALMERÍA \\
\hline BARRANCO & ONIL & ALICANTE \\
\hline BARRANCO & LUCEN & ALMERÍA \\
\hline BARRANCO & BEDAR & ALMERÍA \\
\hline BARRANCO & ALBOX & ALMERÍA \\
\hline BARRANCO & ALBOLODUY & ALMERÍA \\
\hline BARRANCO & BIGASTRO & ALICANTE \\
\hline BARRANCO & MOJÁCAR & ALMERÍA \\
\hline BARRANCO & COCENTAINA & ALICANTE \\
\hline BARRANCO & ARBOLEAS & ALMERÍA \\
\hline BARRANCO & CALASPARRA & MURCIA \\
\hline BARRANCO ALFARERÍAS & ALBOX & ALMERÍA \\
\hline BARRANCO ALTO & MACAEL & ALMERÍA \\
\hline BARRANCO DE AMORÓS & CREVILLENTE & ALICANTE \\
\hline BARRANCO DE FOYETES & BENIDORM & ALICANTE \\
\hline BARRANCO DE LA CAÑADA & LA CAÑADA DE S. URBANO & ALMERÍA \\
\hline BARRANCO DE LA CRUZ & CARBONERAS & ALMERÍA \\
\hline BARRANCO DE LA ESCALERA & ONIL & ALICANTE \\
\hline BARRANCO DE LAS NIEVES & ONIL & ALICANTE \\
\hline BARRANCO DE L'AIGÜERA & BENIDORM & ALICANTE \\
\hline BARRANCO DEL CAÑO & TABERNAS & ALMERÍA \\
\hline BARRANCO DEL CUCO & MACAEL & ALMERÍA \\
\hline BARRANCO GALLEGO & TABERNAS & ALMERÍA \\
\hline BARRANCO HONDO & ALFAZ DEL PI & ALICANTE \\
\hline
\end{tabular}




\begin{tabular}{|c|c|c|}
\hline Calle & Población & Provincia \\
\hline BARRANCO HONDO & LA CAÑADA DE S. URBANO & ALMERÍA \\
\hline BARRANCO HONDO OLLERÍA & BAÑERES & ALICANTE \\
\hline BARRANCO LAS CRUCES & PUERTO LUMBRERAS & MURCIA \\
\hline BARRANCO MIRA & ASPE & ALICANTE \\
\hline BARRANCO MOLAR & SAN JOSÉ ARTESANO & MURCIA \\
\hline BARRANCO MOLAR & ABARÁN & MURCIA \\
\hline BARRANCO MORO & AGUA SALADA & MURCIA \\
\hline BARRANCO PALACIO & ALCALÁ DE LA JOVADA & ALICANTE \\
\hline BARRANCO SAN CAYETANO & CREVILLENTE & ALICANTE \\
\hline BARRANCO SAN JOSÉ & CUEVAS DE ALMANZORA & ALMERÍA \\
\hline BARRANCO, BARRANC & BENIMANTELL & ALICANTE \\
\hline BARRANCO, EL & ASPE & ALICANTE \\
\hline BARRANQUET & BAÑERES & ALICANTE \\
\hline BARRANQUET & ORCHETA & ALICANTE \\
\hline BARRANQUET & BIAR & ALICANTE \\
\hline BARRANQUET & VILLAJOYOSA & ALICANTE \\
\hline BARRANQUET & ALFAZ DEL PI & ALICANTE \\
\hline BARRANQUET & MURO DE ALCOY & ALICANTE \\
\hline BARRANQUET DEL AZAGADOR & AGUAS DE BUSOT & ALICANTE \\
\hline BARRANQUETE & LA ALBERCA & MURCIA \\
\hline BARRANQUETE & ILLAR & ALMERÍA \\
\hline BARRANQUETS & VERGEL & ALICANTE \\
\hline BARRANQUETS, CARRERES & SETLA, MIRARROSA Y MIRAFLOR & ALICANTE \\
\hline BARRANQUET, PASAJE EL & BUSOT & ALICANTE \\
\hline BARRANQUILLO & SAN JOSÉ & ALMERÍA \\
\hline BARRANQUILLO & LUCEN & ALMERÍA \\
\hline BARRANQUILLO & VELEFIQUE & ALMERÍA \\
\hline BARRANQUILLO, EL & TORRE-PACHECO & MURCIA \\
\hline BOCAMINA & FORTUNA & MURCIA \\
\hline BOQUERA & POZO ESTRECHO & MURCIA \\
\hline BOQUERA & PETREL & ALICANTE \\
\hline BOQUERA & CARTAGENA & MURCIA \\
\hline BOQUERA & CREVILLENTE & ALICANTE \\
\hline BOQUERA & ALUMBRES & MURCIA \\
\hline BOQUERA & TORRE-PACHECO & MURCIA \\
\hline BOQUERA DE LA VIRGEN & PUERTO LUMBRERAS & MURCIA \\
\hline BOQUERA DEL CALVARIO & CREVILLENTE & ALICANTE \\
\hline BOQUERA MARCHANTERO & CREVILLENTE & ALICANTE \\
\hline BOQUERAS & MAZARRÓN & MURCIA \\
\hline BOQUERAS & LA UNIÓN & MURCIA \\
\hline BOQUERA CAMINO DE LA & HONDÓN DE LAS NIEVES & ALICANTE \\
\hline BOQUERA, LA & MACISVENDA & MURCIA \\
\hline BOQUERA, LA & CUESTA BLANCA (PERÍN) & MURCIA \\
\hline BOQUERA, LA & PECHINA & ALMERÍA \\
\hline BOQUERÓN & BALERMA & ALMERÍA \\
\hline CAMINO DERRAMADOR & CREVILLENTE & ALICANTE \\
\hline
\end{tabular}




\begin{tabular}{|c|c|c|}
\hline Calle & Población & Provincia \\
\hline CAMINO RAMBLA & CASA DE LAS MONJAS & MURCIA \\
\hline CANALILLO, EL & BALANEGRA & ALMERÍA \\
\hline CAÑADA & ALHAMA DE MURCIA & MURCIA \\
\hline CAÑADA & RICOTE & MURCIA \\
\hline CAÑADA ABAD & LUBRIN & ALMERÍA \\
\hline CAÑADA DE CARDONA & LA CAÑADA DE S. URBANO & ALMERÍA \\
\hline CAÑADA DEL ALGARROBO & ABARÁN & MURCIA \\
\hline CAÑADA DEL ORO & BALERMA & ALMERÍA \\
\hline CAÑADA LOBDAR & ALBOX & ALMERÍA \\
\hline CAÑADA SAN DIEGO & CUEVAS DE ALMANZORA & ALMERÍA \\
\hline CAÑADA VILLEGAS & BALERMA & ALMERÍA \\
\hline CAÑADA VILLEGAS & BALANEGRA & ALMERÍA \\
\hline CAÑADA, LA & CHIRIVEL & ALMERÍA \\
\hline CAÑADA, LA & LAUJAR DE ANDARAX & ALMERÍA \\
\hline CAÑADA, LA & ABANILLA & MURCIA \\
\hline CAÑADA, LA & OLULA DEL RÍO & ALMERÍA \\
\hline CAÑADA, LA & FINES & ALMERÍA \\
\hline CAÑADA, LA & MACAEL & ALMERÍA \\
\hline CAÑADICA & ALEDO & MURCIA \\
\hline CHORRADERO & ABLA & ALMERÍA \\
\hline CHORRADOR, EL & STA. MARÍA DE NIEVA & ALMERÍA \\
\hline CHORRILLO & SERON & ALMERÍA \\
\hline CHORROS & CREVILLENTE & ALICANTE \\
\hline CUEVAS BOQUERA & CREVILLENTE & ALICANTE \\
\hline DERRAMADOR & LA ROMANA & ALICANTE \\
\hline DERRAMADOR II & BENIDORM & ALICANTE \\
\hline DERRAMADOR, Avda. & BENIDORM & ALICANTE \\
\hline DESAGÜE & CAMPOHERMOSO & ALMERÍA \\
\hline EL CHORRICO & MOLINA DE SEGURA & MURCIA \\
\hline MALECÓN & CALASPARRA & MURCIA \\
\hline PARTIDORES & ARCHIVEL & MURCIA \\
\hline PARTIDORES, LOS & LA CAÑADA DE S. URBANO & ALMERÍA \\
\hline RAMBLA & LORCA & MURCIA \\
\hline RAMBLA & PORTMÁN & MURCIA \\
\hline RAMBLA & MOLINA DE SEGURA & MURCIA \\
\hline RAMBLA & BENIAJÁN & MURCIA \\
\hline RAMBLA & POZO ESTRECHO & MURCIA \\
\hline RAMBLA & PALAS & MURCIA \\
\hline RAMBLA & SANTOMERA & MURCIA \\
\hline RAMBLA & LUBRIN & ALMERÍA \\
\hline RAMBLA & LOS GARRES-BENIAJÁN & MURCIA \\
\hline RAMBLA & PULPÍ & ALMERÍA \\
\hline RAMBLA & EL PALMAR & MURCIA \\
\hline RAMBLA & CHURRA (ESPINARDO) & MURCIA \\
\hline RAMBLA & LOS RAMOS-BENIAJÁN & MURCIA \\
\hline RAMBLA & TORREAGÜERA-BENIAJÁN & MURCIA \\
\hline
\end{tabular}




\begin{tabular}{|c|c|c|}
\hline Calle & Población & Provincia \\
\hline RAMBLA & CABEZO DE TORRES & MURCIA \\
\hline RAMBLA & BALERMA & ALMERÍA \\
\hline RAMBLA & GARRUCHA & ALMERÍA \\
\hline RAMBLA & TORRE HORADADA & ALICANTE \\
\hline RAMBLA & ALBUJÓN & MURCIA \\
\hline RAMBLA & YECLA & MURCIA \\
\hline RAMBLA & CORVERA & MURCIA \\
\hline RAMBLA & JAVALÍ NUEVO & MURCIA \\
\hline RAMBLA & CUESTA BLANCA (PERIN) & MURCIA \\
\hline RAMBLA & CARTAGENA & MURCIA \\
\hline RAMBLA & DOLORES (TORRE PACHECO) & MURCIA \\
\hline RAMBLA & TOTANA & MURCIA \\
\hline RAMBLA & ISLA PLANA-PUERTO DE MAZARRÓN & MURCIA \\
\hline RAMBLA & JAVALÍ VIEJO & MURCIA \\
\hline RAMBLA & ALBOLODUY & ALMERÍA \\
\hline RAMBLA & SANTA POLA & ALICANTE \\
\hline RAMBLA & ALGAR & MURCIA \\
\hline RAMBLA & AGUA AMARGA & ALMERÍA \\
\hline RAMBLA & BALANEGRA & ALMERÍA \\
\hline RAMBLA & LOS ALCÁZARES & MURCIA \\
\hline RAMBLA & CUEVAS DE REYLLO & MURCIA \\
\hline RAMBLA & RIBERA DE MOLINA & MURCIA \\
\hline RAMBLA & SANGONERA LA VERDE & MURCIA \\
\hline RAMBLA & EL MIRADOR & MURCIA \\
\hline RAMBLA & RICOTE & MURCIA \\
\hline RAMBLA & ALGEZARES & MURCIA \\
\hline RAMBLA & ALHAMA DE MURCIA & MURCIA \\
\hline RAMBLA ALGEZARES & YECLA & MURCIA \\
\hline RAMBLA ALTA & CALLOSA DE SEGURA & ALICANTE \\
\hline RAMBLA ALTA & LORCA & MURCIA \\
\hline RAMBLA AÑAFLI & TURRE & ALMERÍA \\
\hline RAMBLA BAJA & CALLOSA DE SEGURA & ALICANTE \\
\hline RAMBLA BAJA & LORCA & MURCIA \\
\hline RAMBLA BIZNAGA & CASA DE LAS MONJAS & MURCIA \\
\hline RAMBLA CARRILLO & PECHINA & ALMERÍA \\
\hline RAMBLA CELADOS & ALHAMA DE MURCIA & MURCIA \\
\hline RAMBLA CEMENTERIO VIEJO & TORREAGÜERA-BENIAJÁN & MURCIA \\
\hline RAMBLA CHAPI & AGOST & ALICANTE \\
\hline RAMBLA CHONGA & VILLENA & ALMERÍA \\
\hline RAMBLA CIRERA & CUEVAS DE ALMANZORA & ALMERÍA \\
\hline RAMBLA DE ALMERÍA & PURCHENA & ALMERÍA \\
\hline RAMBLA DE BENITO & ABARÁN & MURCIA \\
\hline RAMBLA DE CALVO SOTELO & CALLOSA DE SEGURA & ALICANTE \\
\hline RAMBLA DE GEVAS & PURCHENA & ALMERÍA \\
\hline RAMBLA DE GRACIA & DALIAS & ALMERÍA \\
\hline RAMBLA DE GRASSO & CHURRA (ESPINARDO) & MURCIA \\
\hline
\end{tabular}




\begin{tabular}{|c|c|c|}
\hline Calle & Población & Provincia \\
\hline RAMBLA DE GRASSO & CABEZO DE TORRES & MURCIA \\
\hline RAMBLA DE HERRERÍAS & GADOR & ALMERÍA \\
\hline RAMBLA DE HIZAR & LAUJAR DE ANDARAX & ALMERÍA \\
\hline RAMBLA DE LA CHANCA & ALMERÍA & ALMERÍA \\
\hline RAMBLA DE LA CHURRA & CHURRA (ESPINARDO) & MURCIA \\
\hline RAMBLA DE LA GITANA & AGUADULCE & ALMERÍA \\
\hline RAMBLA DE LA OLIVERA & CARBONERAS & ALMERÍA \\
\hline RAMBLA DE LA PTA. DE S. GINÉS & LORCA & MURCIA \\
\hline RAMBLA DE LA SANTA & TOTANA & MURCIA \\
\hline RAMBLA DE LAS CRUCES & ADRA & ALMERÍA \\
\hline RAMBLA DE LO VALLEJO & LOS ALCÁZARES & MURCIA \\
\hline RAMBLA DE LOS PEÑONES & LORCA & MURCIA \\
\hline RAMBLA DE MÉNDEZ NÚÑEZ & ALICANTE & ALICANTE \\
\hline RAMBLA DE MOSCOLUX & GADOR & ALMERÍA \\
\hline RAMBLA DE SAN ROQUE & ALHAMA DE MURCIA & MURCIA \\
\hline RAMBLA DEL CARMEN & CABEZO DE TORRES & MURCIA \\
\hline RAMBLA DEL CARMEN & MONTEAGUDO & MURCIA \\
\hline RAMBLA DEL CHARCO & LA CAÑADA DE S. URBANO & ALMERÍA \\
\hline RAMBLA DEL CHARCO & ÁGUILAS & MURCIA \\
\hline RAMBLA DEL HORNILLO & HUERCAL-OVERA & ALMERÍA \\
\hline RAMBLA DEL MOLINO & BARINAS & MURCIA \\
\hline RAMBLA DEL MORO & CIEZA & MURCIA \\
\hline RAMBLA DEL OBISPO ORBERA & ALMERÍA & ALMERÍA \\
\hline RAMBLA DEL POZO & CARBONERAS & ALMERÍA \\
\hline RAMBLA DEL REALEJO & CIEZA & MURCIA \\
\hline RAMBLA DEL SORDO & LA ALBERCA & MURCIA \\
\hline RAMBLA DON DIEGO & ALHAMA DE MURCIA & MURCIA \\
\hline RAMBLA EL ALBIR & ALFAZ DEL PI & ALICANTE \\
\hline RAMBLA GARRIGA & ÁGUILAS & MURCIA \\
\hline RAMBLA HUYTAR MAYOR & OLULA DEL RÍO & ALMERÍA \\
\hline RAMBLA LA QUINTA & FELI & MURCIA \\
\hline RAMBLA LAS CANALAS & LORCA & MURCIA \\
\hline RAMBLA LAS CHATAS & LORCA & MURCIA \\
\hline RAMBLA LAS PERAS & TOTANA & MURCIA \\
\hline RAMBLA LECHUGA & CAÑADA DE S. URBANO & ALMERÍA \\
\hline RAMBLA LOS HUERTOS & LA UNIÓN & MURCIA \\
\hline RAMBLA MELLADO & LORCA & MURCIA \\
\hline RAMBLA SALADA & FORTUNA & MURCIA \\
\hline RAMBLA SAN GINÉS & LORCA & MURCIA \\
\hline RAMBLA SAN JOSÉ & LORCA & MURCIA \\
\hline RAMBLA ZARZAL & ADRA & ALMERÍA \\
\hline RAMBLA ZORRERAS & CARBONERAS & ALMERÍA \\
\hline RAMBLAR & ALHAMA DE MURCIA & MURCIA \\
\hline RAMBLAR, PLAZA DEL & TOTANA & MURCIA \\
\hline RAMBLA, LA & CASAS DEL SEÑOR & ALICANTE \\
\hline RAMBLA, LA & ALFAZ DEL PI & ALICANTE \\
\hline
\end{tabular}




\begin{tabular}{|lll|}
\hline Calle & Población & Provincia \\
\hline RAMBLA, LA & CUEVAS DE ALMANZORA & ALMERÍA \\
RAMBLA, LA & VILLARICOS & ALMERÍA \\
RAMBLA, LA & POZO LOS FRAILES & ALMERÍA \\
RAMBLA, LA & SALINAS & ALICANTE \\
RAMBLA, LA & VILLENA & ALICANTE \\
RAMBLA, LA & ALMENDRICOS & MURCIA \\
RAMBLA, LA & TURRE & ALMERÍA \\
RAMBLA, LA & AGUADULCE & ALMERÍA \\
RAMBLA, LA & CREVILLENTE & ALICANTE \\
RAMBLETA & COX & ALICANTE \\
RAMBLETA & ALMAGROS & MURCIA \\
RAMBLETA & CAÑADA & ALICANTE \\
RAMBLETA & ELCHE & ALICANTE \\
RAMBLETA DEL CONVENTO & JUMILLA & MURCIA \\
RAMBLETA DEL PÓSITO & JUMILLA & MURCIA \\
RAMBLETA, CAMINO DE & GRANJA DE ROCAMORA & ALICANTE \\
RAMBLETA, LA & JIMENADO & MURCIA \\
RAMBLICA & CALASPARRA & MURCIA \\
RAMBLICA, LA & TOTANA & MURCIA \\
RAMBLILLA & BAYARCAL & ALMERÍA \\
RAMBLILLA & ALHAMA DE MURCIA & MURCIA \\
RAMBLILLA & SERON & ALMERÍA \\
RAMBLILLA & CARTAGENA & MURCIA \\
RAMBLILLA & LAS NORIAS & ALMERÍA \\
RAMBLILLA & STA. MARÍA DEL ÁGUILA & ALMERÍA \\
RAMBLILLA DE HÍJAR & LAUJAR DE ANDARAX & MURCIA \\
RAMBLILLA DE TEJERAS & LORCA & MURCIA \\
RAMBLILLA SAN LÁZARO & LORCA & MURCIA \\
RAMBLILLAS, LAS & ALHAMA DE MURCIA & MURCIA \\
RAMBLIZO & EL BOJAR-BENIAJÁN & MURCIA \\
RAMBLIZO & YECLA & MURCIA \\
REGUERO & PEGO & ALICANTE \\
RIHUETE ALTO & PUERTO DE MAZARRÓN & MURCIA \\
SALTADOR, EL & HUERCAL-OVERA & ALMERÍA \\
SALTO DEL AGUA & GADOR & ALMERÍA \\
SANGRADOR & COCENTAINA & ALICANTE \\
SERVIDUMBRES & PUEBLA DE VICAR & ALMERÍA \\
TARQUINA & ABANILLA & MURCIA \\
TORRENTERA & CALASPARRA & \\
\hline
\end{tabular}

